\title{
Choroidal Nevus and Melanoma in Patients with Oculocutaneous Albinism
}

\author{
Pukhraj Rishi Mihir Trivedi Kummamuri Sreelakshmi \\ Shri Bhagwan Mahavir Vitreoretinal Services, Sankara Nethralaya, Chennai, India
}

\section{Established Fact}

- Caucasians have a higher incidence of uveal melanoma than Asians.

- Choroidal nevus and uveal melanoma have been reported in Caucasian patients with oculocutaneous albinism.

\section{Novel Insights}

- This report describes choroidal variegations, nevus, and melanoma in Asian patients with oculocutaneous albinism.

\section{Keywords}

Eye $\cdot$ Tumor $\cdot$ Choroid $\cdot$ Albinism $\cdot$ Nevus $\cdot$ Melanoma

\begin{abstract}
Background: There are limited reports of uveal "melanocytic" lesions in patients with oculocutaneous albinism, with no reports from Asia. Objectives: In this study, we report 3 eyes with uveal "melanocytic" lesions in Asian patients with oculocutaneous albinism. Methods: Retrospective small case series. Three eyes of 2 Asian patients with oculocutaneous albinism were included. Case 1 was a 54-year-old female who presented with juxtapapillary choroidal melanoma and underwent enucleation. Case 2 was a 39-year-old male with
\end{abstract}

\section{KARGER}

(c) 2019 S. Karger AG, Basel

E-Mail karger@karger.com

www.karger.com/oop pigmented choroidal nevus in the right eye and suspicious nevus/choroidal variegation in the left eye. Results: For case 1, metastatic workup including ultrasound of the abdomen, liver function test, and chest X-ray was unremarkable. Magnetic resonance imaging of the cranium ruled out extraocular extension. The left eye was enucleated due to the large tumor size and poor visual potential, and histopathological examination confirmed the diagnosis of choroidal melanoma with mixed cell type. At the last follow-up 6 months after enucleation, there was no evidence of metastasis. Case 2 was observed. Conclusion: Pigmented choroidal nevus and melanoma are rarely observed in patients with oculocutaneous albinism.

(c) 2019 S. Karger AG, Basel 


\section{Introduction}

Although cutaneous malignant melanoma is quite commonly reported in patients with albinism, uveal "melanocytic" lesions are rare. Oculocutaneous albinism (OCA) is the most common form of albinism. Hereby, we report one case each, of pigmented choroidal nevus and choroidal melanoma in patients with OCA. Pubmed search using the keywords albinism, choroidal nevus, and choroidal melanoma revealed four relevant reports. Such cases have never been reported in Asian subjects.

\section{Materials and Methods}

\section{Case 1}

A 54-year-old albinotic Asian Indian female presented with complaints of longstanding photophobia in both eyes and diminished vision in the left eye with headache of 6 months' duration [1]. She had albinotic features of fair skin, and yellow-white hair, eyelashes and eyebrows. She had no personal history of any other malignancy. There was no family history of any malignancy. Bestcorrected visual acuity was 6/36 in the right eye and "perception of light" in the left eye. Horizontal pendular nystagmus and brightred pupillary reflex were noted bilaterally. Fundus examination revealed non-pigmented choroid and retinal pigment epithelium in both eyes through which the choroidal vessels were well appreciated. Foveal hypoplasia was noted in the right eye. The left eye showed a fairly amelanotic juxtapapillary choroidal tumor measuring 10.0 (horizontal basal) $\times 10.8$ (vertical basal) $\times 9.5 \mathrm{~mm}$ (height) in size, with secondary retinal detachment (T3aN0M0). Tumor characteristics on ultrasound scan revealed moderate to low internal reflectivity, acoustic hollowing, and shifting subretinal fluid (Fig. 1). There was no evidence of extraocular extension. A clinical diagnosis of large choroidal melanoma was established in the left eye.

Metastatic workup including ultrasound of the abdomen, magnetic resonance imaging of the cranium, and chest X-ray was unremarkable. Treatment options were discussed and the left eye was enucleated due to the large tumor size/poor visual potential, rather than performing eye-preserving therapy (radiation).

\section{Case 2}

A 39-year-old Asian male patient presented with a history of low vision in both eyes since childhood. He had albinotic features of hypopigmented skin, eyelashes, hair and eyebrows with no pertinent family history. Best-corrected visual acuity was $6 / 60$ bilaterally. He had no personal history or family history of any malignancy. Bright red pupillary reflex with horizontal jerk nystagmus was noted. Fundus examination revealed albinotic features with $3 \times 1.5 \mathrm{~mm}$ choroidal nevus in the right eye and $1 \times 1.5 \mathrm{~mm}$ choroidal variegation in the left eye (Fig. 2).

\section{Results}

For the enucleated eye, histopathological examination confirmed the diagnosis of choroidal melanoma with mixed cell type. At the last follow-up 6 months after enucleation, there was no evidence of systemic metastasis.

\section{Discussion}

OCA is a heterogenous group of autosomal recessive disorders affecting melanin synthesis, characterized by congenital hypopigmentation of the skin, hair, and eyes. Reduced visual acuity, photophobia, iris transillumination, foveal hypoplasia, nystagmus, and an abnormal decussation of nerve fibers at the optic chiasm are common features. OCA is divided into 5 categories $(1 \mathrm{a}, 1 \mathrm{~b}, 2,3,4)$ according to clinical features and tyrosinase gene activity. Type 1a OCA lacks tyrosinase activity (tyrosinase-negative) and displays complete absence of melanin production. Types 1b, 2, 3 manifest a spectrum of tyrosinase activity (tyrosinase-positive). OCA4 is characterized by physical features that are similar to those of OCA2. OCA4 is associated with mutations in the SLC45A2 gene (also called the membrane-associated transporter protein; MATP). Cutaneous melanocytic nevi appear in types $1 \mathrm{~b}$, 2,3 , and 4 [2].

Very few cases of choroidal melanoma and choroidal nevus have been reported in patients with OCA. Choroidal melanoma is even rarer in Asians [3]. Theoretically, the incidence of choroidal melanoma should increase in patients with pale irides with intermittent exposure to ultraviolet light. Pale irides have a lesser ability to block sunlight than the dark irides. The higher incidence of inferior pole melanoma where the greatest incidence of incident light impinges further supports this hypothesis. An alternate hypothesis is that the locus at 15q13.1 determines pigmentation of eyes and skin by regulating the expression of $O C A 2$, which codes for $P$ protein involved in melanin synthesis. The allele associated with darker eye color (allele T), associated with a uveal melanoma-protective effect, enhances OCA2 expression resulting in darkly pigmented melanocytes. The $\mathrm{C}$-allele conversely reduces expression of OCA2 producing lightly colored melanocytes. This supports the hypothesis that genetic determinants of dark eye pigmentation are protective while lighter pigmentation alleles confer a risk effect on uveal melanoma [1]. 

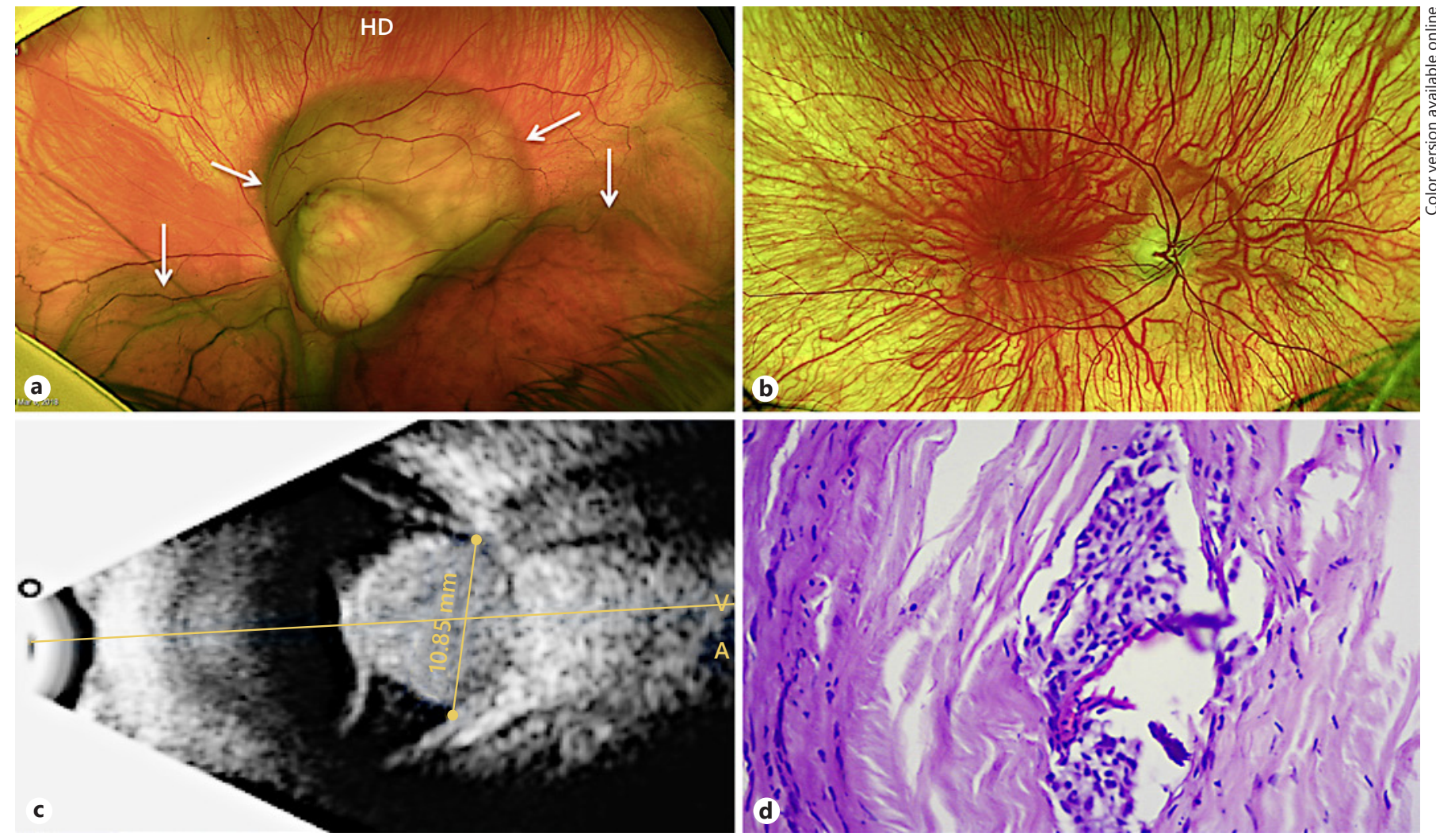

Fig. 1. Wide-angle fundus image showing albinotic fundus (a) and fairly amelanotic choroidal melanoma at the posterior pole (oblique arrows) with inferior choroidal detachment (vertical arrows). The fellow eye shows albinotic fundus (b) without melanocytic lesions. Right eye ultrasound reveals dome-shaped choroidal melanoma measuring $10.0 \times 10.8 \times 9.5$ with moderate-to-low internal reflectivity, acoustic hollowing, and exudative retinal detachment (c). d Histopathology microphotograph shows mixed cell type of the choroidal melanoma (H\&E, $\times 20)$.
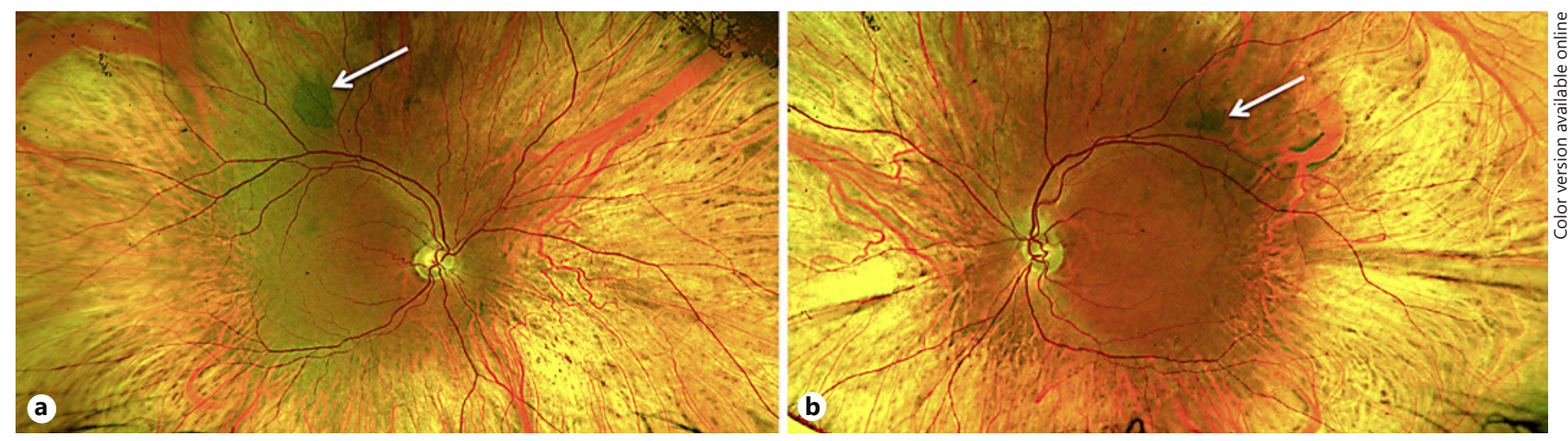

Fig. 2. Wide-angle fundus photograph showing albinotic fundus with pigmented choroidal nevus seen in right eyes (a; arrow) and choroidal variegation in the left eye (b; arrow). 
The rarity of reported cases of choroidal melanoma in albinos raises a variety of questions. The possibility exists that many more cases have occurred but are not reported. However, if melanomas were present, it can be presumed that they would be found and reported. This argues that the number of reported cases is reflective of the true incidence of choroidal melanoma in albinos, which at the very least is not markedly elevated over that of the general population. Realistically though, it is difficult to estimate the incidence of uveal melanoma in an orphan disease like OCA. Casswell et al. [4] reported a case of amelanotic choroidal melanoma in a 22 -year-old female with tyrosinase-positive OCA. It was the first case to be reported with this uncommon association. As there was an associated retinal detachment with a large tumor, the eye was enucleated. Kheterpal et al. [5] reported a 68-year-old black albino man with poor visual acuity and an amelanotic choroidal melanoma, confirmed cytologically and treated with a unilateral choroidal nevus in an 8-year-old girl with OCA. Our cases were similar to that published in the literature, although no such reports have been published from Asia. Perhaps it would be interesting to know more about the subtype of OCA in patients with reported nevi/uveal melanoma. This requires further biochemical and genetic testing.

\section{Conclusion}

Our cases highlight a rare occurrence of pigmented choroidal melanoma and choroidal nevi in patients with OCA. Further studies are required to explore the role of ocular pigmentation genes in the etiology of uveal nevi and melanoma, especially in Asians where there is a lower risk of developing these conditions. plaque radiotherapy. Sharma et al. [6] reported a case of

\section{Statement of Ethics}

Subjects (or their parents or guardians) have given their written informed consent. The study protocol has been approved by the research institute's committee on human research.

\section{Disclosure Statement}

The authors have no conflicts of interest to declare.

\section{Funding Sources}

There are no funding sources to declare.

\section{Author Contributions}

P.R.: Conceptualization, data analysis, writing, editing and revising the manuscript.

M.T.: Data gathering and data analysis, writing, editing and revising the manuscript.

K.S.: Data gathering and data analysis, editing and revising the manuscript.

\section{References}

1 Rishi P, Shields CL, Khan MA, Patrick K, Shields JA. Headache or eye pain as the presenting feature of uveal melanoma. Ophthalmology. 2013 Sep;120(9):1946-7.e2.

2 Bakos RM, Argenziano G, Zalaudek I, Masiecopy of pigmented melanocytic nevi in patients with oculocutaneous albinism. J Am Acad Dermatol. 2009 Mar;60(3):487-9.

3 Dhupper M, Biswas J, Gopal L, Kumar SK, Khetan V. Clinicopathological correlation of choroidal melanoma in Indian population: A study of 113 cases. Oman J Ophthalmol. 2012 Jan;5(1):42-5.

4 Casswell AG, McCartney AC, Hungerford JL. Choroidal malignant melanoma in an albino. Br J Ophthalmol. 1989 Oct;73(10):840-5.

5 Kheterpal S, Shields JA, Shields CL, De Potter $\mathrm{P}$, Ehya H, Eng KY. Choroidal melanoma in an African-American albino. Am J Ophthalmol. 1996 Dec;122(6):901-3.

6 Sharma P, Kaliki S, Peña MS, Shields CL. Pigmented choroidal nevus in a child with oculocutaneous albinism. J AAPOS. 2013 Apr; 17(2):203-5. ro NC, Zoratto G, Cartell A, et al. Dermatos- 